\title{
Unitary and Non-Unitary Representations of the Complex Inhomogeneous Lorentz Group*
}

\author{
ERIC H. RofFman \\ Courant Institute of Mathematical Sciences, New York University \\ Received June 5, 1966
}

\begin{abstract}
We construct unitary and non-unitary representations of the complex inhomogeneous Lorentz group, including all its unitary, irreducible representations. We discuss the decomposition of these representations when they are restricted to the real inhomogeneous Lorentz group. We also discuss the representations of the Poincaré group for which the translation subgroup transforms under a not necessarily unitary representation. We summarize briefly the physical motivation for this study.
\end{abstract}

\section{Introduction}

This is the first of (at least) two articles on the structure and possible applications to scattering theory and particle physics of the complex inhomogeneous Lorentz group, CILG. In this article we discuss the representations of the group. We do not restrict ourselves to unitary representations. Although we say nothing about irreducibility or "completeness" of the non-unitary representations, we do find all the unitary, irreducible representations of CILG.

In the next article we plan to discuss some possible applications and the physical interpretation of CILG.

We begin in Section II with a series of definitions, for the purpose of naming the various objects we will construct in later sections. We believe that a comparison of the general structure with the particular examples given, will clarify the constructions for the reader. We then define the CILG and several of its important subgroups in Section III. The method of induced representations, which we will use in finding the representations of CILG, requires us to find the "sesquilinear system" representations of certain subgroups of CILG: the "little" groups $\mathcal{B}$ and $S L(2 C)$ (Sections IV and V), and the translation group, $T$ (Section VI). Finally, we put the results together to find the representations of CILG in Section VII, and of its physical subgroup $\mathscr{P}$, (the Poincaré group) in Section VIII.

In Section $\mathrm{V}$, which reviews the representation theory of $S L(2 C)$, we derive the asymptotic form of a certain operator. In Section VIII, we discuss how representations of $\mathscr{P}$ are contained in the representations

* Supported by the National Science Foundation (NSF-GP-58) while at Brandeis University and by the National Science Foundation, Grant NSF-GP-3465, while at the Courant Institute of Mathematical Sciences.

17 Commun. math. Phys., Vol. 4 
of CILG. In the final Section, IX, we indicate briefly how these results are connected to the physical interpretation of CILG, and its application to complex angular momentum.

\section{Representations in topological spaces}

For not necessarily unitary representations of non-compact groups, the machinery of finite dimensional vector spaces, which was appropriate for irreducible, unitary representations of compact and abelian groups, no longer suffices. Indeed, we now need to consider representations in linear topological spaces which, if they are Hilbert spaces at all, are infinite dimensional. In general, they will not even be in Banach spaces ${ }^{1}$.

In order to have an invariant sesquilinear form, we consider pairs consisting of a space $D$ and its dual $D^{\prime}$, with the representation in $D^{\prime}$ being the adjoint of the inverse representation in $D$.

We shall introduce these sesquilinear systems, then define induced representations. The theory of induced representations, using sesquilinear systems, will be our main tool in constructing representations of CILG.

Def. A sesquilinear system, SLS, is a pair of complex linear topological spaces $D_{1}$ and $D_{2}$, and a sesquilinear (linear - antilinear) form

such that

$$
(,): D_{1} \otimes D_{2} \rightarrow \mathbb{C}
$$

$$
\begin{aligned}
\left(\alpha_{1} \xi_{1}+\alpha_{2} \xi_{2}, \beta_{1} \eta_{1}+\beta_{2} \eta_{2}\right)= & \bar{\alpha}_{1} \beta_{1}\left(\xi_{1}, \eta_{1}\right)+\bar{\alpha}_{2} \beta_{1}\left(\xi_{2}, \eta_{1}\right)+ \\
& +\bar{\alpha}_{1} \beta_{2}\left(\xi_{1}, \eta_{2}\right)+\bar{\alpha}_{2} \beta_{2}\left(\xi_{2}, \eta_{2}\right),
\end{aligned}
$$

the definition of sesquilinearity, and

where

$$
\begin{array}{lll}
\left(\xi, D_{2}\right)=0 & \text { iff } & \xi=0 \\
\left(D_{1}, \eta\right)=0 & \text { iff } & \eta=0
\end{array}
$$

$$
\begin{gathered}
\xi, \xi_{1}, \xi_{2} \in D_{1} \text { and } \eta, \eta_{1}, \eta_{2} \in D_{2} \\
\alpha_{1}, \alpha_{2}, \beta_{1}, \beta_{2} \in \mathbb{C} .
\end{gathered}
$$

Def. Let $D_{1}$ and $D_{2}$ be the linear spaces of an SLS. Let $E_{1}$ and $E_{2}$ be the spaces of endomorphisms of $D_{1}$ into itself and $D_{2}$ into itself, respectively.

A homomorphism

$$
\begin{aligned}
& G \rightarrow E_{1} \otimes E_{2} \\
& g \rightarrow T_{1}(g) \otimes T_{2}(g)
\end{aligned}
$$

1 A discussion of this point, as well as the most complete discussion of nonunitary representations that exists, to my knowledge, in the mathematical literature, is given in reference [1]. See also references [2], [4], [5] and [6] for the material of this section. 
of $G$ into $E_{1} \otimes E_{2}$ is called an SLS representation if for all $\xi \in D_{1}, \eta \in D_{2}$, $g \in G$

or equivalently

$$
\left(T_{1}(g) \xi, T_{2}(g) \eta\right)=(\xi, \eta)
$$

$$
\left(T_{1}(g) \xi, \eta\right)=\left(\xi, T_{2}\left(g^{-1}\right) \eta\right)
$$

There are fairly satisfactory notions of equivalence and irreducibility for linear system representations, but they require much more detailed topological and algebraic considerations than we wish to discuss here ${ }^{2}$.

Def. A $G$ space is a triple, $(G, X, \Phi)$, consisting of a topological group $G$ and a topological space $X$, with a map $\Phi$ called the action,

$$
\begin{gathered}
\Phi: G \otimes X \rightarrow X \\
\Phi(g, x)=x_{g},
\end{gathered}
$$

continuous in both $G$ and $X$, such that

$$
\begin{gathered}
x_{e}=x, \quad x \in X \\
\left(x_{g_{1}}\right)_{g_{2}}=x_{g_{1} g_{2}}, \quad x_{g_{2}}, x_{g_{1} g_{2}} \in X, g_{1}, g_{2} \in G .
\end{gathered}
$$

If the action of $G$ on $X$ is transitive, which means that for every $x, y \in X$ there is a $g \in G$ such that

$$
y=x_{g},
$$

then $X$ is called a transitive $G$-space, or a homogeneous space.

Def. An SLS function space $\mathfrak{F}$, over a space $X$, is an SLS together with a space $K_{\mathfrak{F}}$ of functions

$$
k: X \rightarrow D_{1} \otimes D_{2}, \quad k \in K_{\mathscr{F}} .
$$

For every $k$ in an SLS function space, there is another function $\hat{k}$ defined by

$$
\hat{k}: X \rightarrow \mathbb{C}
$$

$$
\hat{k}(x)=\left(k_{1}(x), k_{2}(x)\right) \equiv\left(k_{1}, k_{2}\right)(x) \quad k_{1} \in D_{1}, k_{2} \in D_{2} .
$$

Suppose that the space $X$ is a $G$-space with action $\Phi(g, x)=x_{g}$. Then we define

Correspondingly,

$$
k_{g}(x)=k\left(x_{g}\right)
$$

$$
\hat{k}_{g}(x)=\hat{k}\left(x_{g}\right) \text {. }
$$

Def. If $d \mu_{x}$ is an invariant measure on a $G$-space $X$, and $\mathfrak{F}$ is an SLS function space, then the space of all functions $k \in K_{\mathscr{F}}$ such that

$$
\int_{X} \hat{k}(x) d \mu_{x} \equiv \int\left(k_{1}(x), k_{2}(x)\right) d \mu_{x}
$$

${ }^{2}$ See reference [1], also reference [2]. 
exists and is finite, with the representation

$$
T(g) k=k_{g}
$$

is called a functional representation space of $G$.

Obviously, then

$$
\int_{X} \hat{k}(x) d \mu_{x}=\int_{X} \hat{k}\left(x_{g}\right) d \mu_{x}=\int_{X} \hat{k}_{g}(x) d \mu_{x} .
$$

Note that if the SLS is a Hilbert space with its natural dual, and the function space based on it consists of all integrable functions (for some measure on $X$ ) from $X$ into $\mathscr{H} \otimes \mathscr{H}$, then the SLS function space is a direct integral Hilbert space.

Def. Let $H$ be a closed subgroup of $G$. Let $R_{I I}$ be an SLS representation of $H$ based on the SLS $D_{1}, D_{2},(,)_{D}$. Let $\mathfrak{F}$ be a functional representation space of $G$, and suppose that $K$ contains all functions such that

satisfies

$$
f: G \rightarrow D_{1} \otimes D_{2}
$$

$$
\begin{gathered}
f_{1}(\xi g)=T_{1}(\xi) f_{1}(g) \\
f_{2}(\xi g)=T_{2}(\xi) f_{2}(g) \\
\xi \in H, g \in G, f \in K_{\xi} .
\end{gathered}
$$

In each $G / H$ coset we pick a representative $k \in G$. Let $\Psi$ be the set of all $\Psi \in K_{\mathscr{F}}$ such that

$$
\int_{G / H} \hat{\Psi}(k) d \mu_{k} \quad \text { exists and is finite, }
$$

where $d \mu_{k}$ is an invariant measure in $G / H$. Then $\Psi$ is the space of an induced SLS representation, in which the representation is defined by

$$
T(g) \Psi=\Psi_{g} \text {. }
$$

Clearly, the sesquilinear form satisfies

for

$$
\int \hat{\Psi}(k) d \mu_{k}=\int \hat{\Psi}_{g}(k) d \mu_{k}
$$

$$
\begin{aligned}
& \int\left(\Psi_{1}, \Psi_{2}\right)(k) d \mu_{k} \rightarrow \int\left(\Psi_{1}, \Psi_{2}\right)(k g) d \mu_{k} \\
& \quad=\int\left(T_{1}(\xi) \Psi_{1}, T_{2}(\xi) \Psi_{2}\right)\left(k^{\prime}\right) d \mu_{k} \\
& \quad=\int\left(\Psi_{1}, \Psi_{2}\right)\left(k^{\prime}\right) d \mu_{k^{\prime}}
\end{aligned}
$$

where $\xi=k g k^{\prime-1} \in H$ and $k^{\prime} \in G$ is the representative of a coset in $G / H$.

\section{The group CILG}

We discuss the representation of the covering group of the connected component of the complex inhomogeneous Lorentz group. We call this group CILG. 
To define CILG precisely, we need both the additive and the multiplicative structure of two-by-two complex matrices. Hence, we define $G L(2 C)$ to mean the ring of all two-by-two complex matrices and $S L(2 C) \subset G L(2 C)$ to mean the subring of elements which have unit determinant.

We can realize an element of CILG by the triple

$$
\begin{gathered}
(X, \Sigma, \Lambda) \\
X \in G L(2 C) \\
\Sigma, \Lambda \in S L(2 C),
\end{gathered}
$$

with the multiplication law

where

$$
\left(X^{\prime}, \Sigma^{\prime}, \Lambda^{\prime}\right)\left(X^{\prime \prime}, \Sigma^{\prime \prime}, \Lambda^{\prime \prime}\right)=(X, \Sigma, \Lambda)
$$

$$
\begin{aligned}
& X=X^{\prime}+\Sigma^{\prime} X^{\prime \prime} \Lambda^{\prime *} \\
& \Sigma=\Sigma^{\prime} \Sigma^{\prime \prime} \\
& \Lambda=\Lambda^{\prime} \Lambda^{\prime \prime} .
\end{aligned}
$$

(* denotes Hermitian conjugation.)

An equivalent realization, which we need in Section VII, is obtained by setting

The multiplication becomes

$$
\begin{aligned}
& \Gamma=\Sigma A^{*} \\
& \Pi=\Lambda .
\end{aligned}
$$

$$
\left(X^{\prime}, \Gamma^{\prime}, \Pi^{\prime}\right)\left(X^{\prime \prime}, \Gamma^{\prime \prime}, \Pi^{\prime \prime}\right)=(X, \Gamma, \Pi)
$$

where

$$
\begin{aligned}
& X=X^{\prime}+\Gamma^{\prime} \Pi^{\prime *-1} X^{\prime \prime} \Pi^{\prime *} \\
& \Gamma=\Gamma^{\prime} \Pi^{\prime *-1} \Gamma^{\prime \prime} \Pi^{\prime *} \\
& \Pi=\Pi^{\prime} \Pi^{\prime \prime} .
\end{aligned}
$$

The translations are the subgroup

$$
\left(X^{\prime}, 1,1\right)\left(X^{\prime \prime}, 1,1\right)=\left(X^{\prime}+X^{\prime \prime}, 1,1\right) \text {. }
$$

Any $X \in G L(2 C)$ can be written

$$
X=X_{\mu} \sigma^{\mu}=X_{0}-\mathbf{X} \cdot \boldsymbol{\sigma} .
$$

This defines a one-to-one correspondence between $X$ and $X_{\mu}$. Given $X_{\mu}$, we define $X_{+}$to be

$$
X_{0}+\mathbf{X} \cdot \boldsymbol{\sigma} \equiv X_{+} \cdot
$$

$X_{\mu}=X_{\mu}^{1}+i X_{\mu}^{2}$ is a complex four-vector, and $\sigma^{\mu}$ are the Hermitian Pauli matrices.

The metric is

$$
\begin{aligned}
\left\langle\langle X\rangle^{2}=X_{\mu} X^{\mu}\right. & =\left(X^{1}\right)_{\mu}\left(X^{1}\right)^{\mu}-\left(X^{2}\right)_{\mu}\left(X^{2}\right)^{\mu}+2 i\left(X^{1}\right)_{\mu}\left(X^{2}\right)^{\mu} \\
& =\frac{1}{2} \operatorname{Tr}\left(X X_{+}\right) .
\end{aligned}
$$


The map

$$
\begin{aligned}
X & \rightarrow \Sigma X \Lambda^{*} \\
\text { (or } X & \rightarrow \Gamma \Pi^{*-1} X \Pi^{*} \text { ) }
\end{aligned}
$$

leaves $\langle\langle X\rangle\rangle^{2}$ invariant. The pair $(-\Sigma,-\Lambda)$ (respectively $\left.(\Gamma,-\Pi)\right)$ produces the same effect as the pair $(\Sigma, \Lambda)$ (respectively $(\Gamma,+\Pi)$ ). If $(-)$ denotes the equivalence of such pairs then

$(\Sigma, \Lambda) /(-) \cong$ connected component of the complex Lorentz group.

The group $\mathscr{P}$, which is the covering group of the connected component of the Poincaré group, is the group of triples

$$
\left(X^{1}, \Lambda, \Lambda\right)
$$

in the first realization of CILG, and

$$
\left(X^{1}, \Pi \Pi *, \Pi\right)
$$

in the second realization of CILG. $X^{1}$ is a Hermitian matrix. Clearly $\mathscr{P} \subset$ CILG.

\section{The group 3}

We construct unitary and non-unitary representations of the group, which we call 8, which arises as a little group for "zero mass" in the representation theory of CILG.

The general element $\zeta=[\lambda, z, w]$ of $B$ may be written as a pair of matrices

$$
\left[\left(\begin{array}{cc}
\lambda & 0 \\
z & \lambda-1
\end{array}\right),\left(\begin{array}{ll}
\bar{\lambda}-1 & 0 \\
\bar{w} & \bar{\lambda}
\end{array}\right)\right]=[\lambda, z, w]=\zeta
$$

where $\lambda, z$, and $w$ are complex numbers, and the multiplication law is

$$
\begin{aligned}
{\left[\lambda^{\prime}, z^{\prime}, w^{\prime}\right]\left[\lambda^{\prime \prime}, z^{\prime \prime}, w^{\prime \prime}\right] } & =[\lambda, z, w] \\
\lambda & =\lambda^{\prime} \lambda^{\prime \prime} \\
z & =\lambda^{\prime \prime} z^{\prime}+\lambda^{\prime-1} z^{\prime \prime} \\
w & =\lambda^{\prime \prime}-1 w^{\prime}+\lambda^{\prime} w^{\prime \prime} .
\end{aligned}
$$

Notice that

$$
\left[1, z^{\prime}, w^{\prime}\right]\left[1, z^{\prime \prime}, w^{\prime \prime}\right]=\left[1, z^{\prime}+z^{\prime \prime}, w^{\prime}+w^{\prime \prime}\right]
$$

and that for any $\zeta=\left[\lambda, z^{\prime}, w^{\prime}\right]$

$$
\zeta[1, z, w] \zeta^{-1}=\left[1, \lambda^{-2} z, \lambda^{2} w\right] .
$$

The subgroup of all elements of the form $[1, z, w]$ is an invariant, abelian subgroup $T$, isomorphic to the two complex dimensional translation group. 
The irreducible representations of $T$ are labelled by four complex numbers $P_{i}, Q_{i}, i=1,2$, and are defined by

$$
T_{P_{i}, Q_{i}}(1, z, w)\left|P_{i}, Q_{i}\right\rangle=e^{i\left\{z P_{1}+\overline{z Q_{1}}+w P_{2}+\overline{w Q_{2}}\right\}}\left|P_{i}, Q_{i}\right\rangle .
$$

This representation is unitary when $P_{1}=Q_{1}$ and $P_{2}=Q_{2}$.

We can define an action of the whole group on this space $\widetilde{T}$ of irreducible representations of $T$ by

$$
\begin{aligned}
T_{\left[P_{i}, Q_{i}\right]_{\zeta}}(1, z, w) & =T_{P_{i}, Q_{i}}\left[(\zeta)(1, z, w)\left(\zeta^{-1}\right)\right] \\
& =T_{P_{i}, Q_{i}}\left(1, \lambda^{-2} z, \lambda^{2} w\right) .
\end{aligned}
$$

This can be interpreted as

$$
T_{\left[P_{1} P_{2} Q_{1} Q_{2}\right] \zeta}=T_{\lambda^{-2} P_{1}, \lambda^{-2} Q_{1}, \lambda^{2} P_{2}, \lambda^{2} Q_{2}} .
$$

The quantities

$$
\frac{Q_{1}}{P_{1}}=A, \quad P_{1} P_{2}=B, \quad \text { and } \quad \frac{Q_{2}}{P_{2}}=C \quad A, B, C \in \mathbb{C}
$$

are invariants of this action. $B$ is an invariant of an irreducible representation of $3 . A$ and $C$ measure the strength of non-unitary representations.

We now use these results to construct the irreducible representations of 8 , by the method of WIGNER and MACKEY.

$A$. Suppose $P_{i}=Q_{i}=0$. The representation is given by $T(\zeta)=T_{z}(\lambda)$ where $T_{\chi}(\lambda)$ denotes an irreducible representation of the multiplicative group of complex numbers. These are labelled by a pair of complex numbers $\left(n_{1}, n_{2}\right)$, (denoted collectively by $\chi$ ) whose difference is an integer $n$ and whose sum is an arbitrary complex number $\varrho$.

$$
\chi=\left(n_{1}, n_{2}\right)=\left(\frac{\varrho+n}{2}, \frac{\varrho-n}{2}\right) \leftrightarrow\langle\varrho ; n\rangle .
$$

The representation is defined by

$$
T(\lambda, z, w)\left|n_{1}, n_{2}\right\rangle=\lambda^{n_{1}-1} \bar{\lambda}^{n_{3}-1}\left|n_{1}, n_{2}\right\rangle=|\lambda|^{\varrho-2} e^{\text {in [arg } \lambda]}|\varrho, n\rangle .
$$

$B$. Suppose some $P_{i}$ or $Q_{i}$ is not zero. Let it be $P_{1}$. Identify $P_{1}$ with the multiplicative group of complex numbers, i.e. with the subgroup $\Lambda=\{(\lambda, 0,0)\}$.

Let $f: B \rightarrow \widetilde{T}$ have the property for all $\zeta \in \mathcal{B}$, that

$$
f[(1, z, w) \zeta]=e^{i\left\{z P_{1}+\overline{z Q_{1}}+w P_{2}+\overline{w Q_{2}}\right\}} f(\zeta)
$$

where $P_{1}=1, Q_{1}=A, P_{2}=B, Q_{2}=B C$ and $f(\lambda, 0,0)= \pm f(-\lambda, 0,0)$.

The representation is defined on a suitably restricted (in terms of their integrability and asymptotic properties as $\lambda \rightarrow \infty$ ) class of such functions, and is given by

$$
\left[T\left(\zeta^{\prime}\right) f\right](\zeta)=f\left(\zeta \zeta^{\prime}\right)
$$


An irreducible representation is denoted by $A, B, C$ and \pm .

We can make this into an SLS functional representation space of 8 in the following way.

For every $P_{i}, Q_{i}$ we define an SLS by

$$
\begin{array}{lll}
D_{1}=\left\{\alpha\left|P_{i}, Q_{i}\right\rangle\right\} & \text { where } \quad \alpha \in \mathbb{C} \\
D_{2}=\left\{\beta\left|Q_{i}, P_{i}\right\rangle\right\} & \text { where } \quad \beta \in \mathbb{C} .
\end{array}
$$

The sesquilinear form is defined by

$$
\left(\beta\left|Q_{i}, P_{i}\right\rangle, \alpha\left|P_{i}, Q_{i}\right\rangle\right)=\alpha \bar{\beta} .
$$

We define an SLS representation of $\{(1, z, w)\}$ by

$$
T(z, w)\left|P_{i}, Q_{i}\right\rangle=e^{i\left(P_{1} z+\overline{Q_{1} z}+P_{2} w+\overline{\left.Q_{2} w\right)}\right.}\left|P_{i}, Q_{i}\right\rangle
$$

correspondingly

$$
T(z, w)\left|Q_{i}, P_{i}\right\rangle=e^{i\left(Q_{1} z+\widetilde{P_{1} z}+Q_{2} w+\widetilde{\left.P_{2} w\right)}\right.}\left|Q_{i}, P_{i}\right\rangle .
$$

Clearly

$$
\left(\left|Q_{i} P_{i}\right\rangle,\left|P_{i} Q_{i}\right\rangle\right)=\left(T(z, w)\left|Q_{i} P_{i}\right\rangle, T(z, w)\left|P_{i} Q_{i}\right\rangle\right)
$$

hence this is an SLS representation.

The induced SLS representation is defined by equations of the form (4.10) and (4.11), except the range of the functions is an SLS representation space, rather than simply the right or left hand $D_{i}$-space as in (4.10).

\section{SL (2C)}

In this section we review the representation theory of $S L(2 C)^{3}$.

The representation can be defined to act in various spaces. We can choose the space on which the representation acts to have the following properties:

1. There is a representation for each $\chi=\left(n_{1}, n_{2}\right)$, where $n_{1}$ and $n_{2}$ are complex numbers whose difference is an integer. Let the space of functions on which $T_{\chi}$ acts be denoted by $D_{\chi}$.

2. An element $f_{x}$ of $D_{x}$ depends independently on $z$ and $\bar{z}$, it is single valued on the complex $(z, \bar{z})$ plane, and is infinitely differentiable in $z$ and $\bar{z}$.

3. $\hat{f}(z) \equiv z^{n_{1}-1} \bar{z}^{n_{2}-2} f\left(-\frac{1}{z}\right)$ is also infinitely differentiable in $z$ and $\bar{z}$, and single valued.

4. Asymptotically, as $z \rightarrow \infty, f(z) \rightarrow z^{n_{1}-1} \bar{z}^{n_{2}-1} \times$ (const.).

5. The topology of $D_{x}$ is given by the following:

${ }^{3}$ See reference [2]. Reference [8] also has useful information. 
A sequence $f_{m}(z)$ is said to converge to zero in $D_{\chi}$ if in every finite region of the $(z, \bar{z})$ plane and for all $n$, the sequences $f_{m}^{(n)}(z)$ and $f_{m}^{(n)}(z)$ of the $n^{t h}$ derivatives of $f_{m}(z)$ and $\hat{f}_{m}(z)$ converge to zero.

6. $D_{z}$ is complete in this topology.

In this space, the representation is defined by

$$
\begin{gathered}
{\left[T_{\chi}\left(\begin{array}{ll}
\alpha & \beta \\
\gamma & \delta
\end{array}\right) f\right](z)=} \\
(\beta z+\delta)^{n_{1}-1} \overline{(\beta z+\delta)^{n}}-1 f\left(\frac{\alpha z+\gamma}{\beta z+\delta}\right) \\
\left(\begin{array}{ll}
\alpha & \beta \\
\gamma & \delta
\end{array}\right) \in S L(2 C) .
\end{gathered}
$$

It is sometimes convenient to use the notation

$$
\chi=\langle\varrho, n\rangle \text { instead of } \chi=\left(n_{1}, n_{2}\right)
$$

where $\varrho=n_{1}+n_{2}$ and $n=n_{1}-n_{2} . n$ by assumption is an integer. $\varrho$ is an arbitrary complex number.

An "integer point" is a value of $\chi$ for which $n_{1}$ and $n_{2}$ are integers of the same sign, or $\varrho$ is an integer larger in magnitude than $n$. A "real point" is a value of $\chi$ for which $\varrho$ is real, and an "imaginary point" one for which $\varrho$ is purely imaginary.

We define $\bar{\chi}$ as $\left(\bar{n}_{2}, \bar{n}_{1}\right)$ or $\langle\bar{\varrho},-n\rangle$ (because if $f \in D_{z}$, then $\bar{f} \in D_{\bar{\chi}}$ with this definition) and $-\chi$ as $\left(-n_{1},-n_{2}\right)$ or $\langle-\varrho,-n\rangle$.

If $\chi$ is an integer point and $\varrho$ is positive then there is a finite dimensional invariant subspace $E_{\chi}$ in $D_{\chi}$, which transforms according to a spinor representation.

If $\chi$ is an integer point and $\varrho$ is negative then there is an infinite dimensional invariant subspace $F_{\chi}$ in $D_{\chi}$, and $D_{x} / F_{\chi}$ transforms according to a spinor representation.

Two representations $\chi$ and $\chi^{\prime}$ are equivalent if neither is an integer point and either

or

$$
\chi=\chi^{\prime}
$$

$$
\chi=-\chi^{\prime} \text {. }
$$

If $\chi$ is a positive integer point, i.e. $\varrho>n>0$, then

and

$$
D_{\langle\varrho, n\rangle} \mid E_{\langle\varrho, n\rangle} \cong D_{\langle n, \varrho\rangle} \cong D_{\langle-n,-\varrho\rangle} \cong F_{\langle-\varrho,-n\rangle}
$$

$$
E_{\langle\varrho, n\rangle} \cong D_{\langle-0,-n\rangle} \mid F_{\langle-\varrho,-n\rangle} .
$$

The representation in the space $D_{\chi}$ is unitary if $\chi$ is an imaginary point (the primary series of representations) or if $\chi$ is a real point for which $|\varrho|<2$ and $n=0$ (supplementary series of representations).

If $\varrho=2, n=0$, then the finite dimensional subspace $E_{\langle 2,0\rangle}$ transforms according to the identity representation, which is unitary. The identity representation is the only unitary representation which is finite dimensional. 
One can also define the representation on a space $B_{z}$ of complexvalued functions whose domain is $S U(2)^{4}$.

For every representation $g \rightarrow T_{\chi}(g)$ on $B_{\chi}$, there is another representation defined on $B_{-\bar{x}}$

$$
g \rightarrow T_{-\bar{\chi}}(g)
$$

such that there is an invariant sesquilinear form $S$ on this pair of spaces:

namely,

$$
\begin{gathered}
S: B_{\chi} \otimes B_{-\bar{x}} \rightarrow \mathbb{C} \\
S(f, g)=S\left(T_{-\bar{z}}(g) f, T_{z}(g) k\right)
\end{gathered}
$$

$$
S(f, g)=\int \overline{f(u)} g(u) d u
$$

where $f \in B_{-\bar{x}} k \in B_{x}$, and $d u$ is Haar measure for $S U(2)$.

When we restrict ourselves to $u \in S U(2) \subset S L(2 C)$ the representations

$$
u \rightarrow T_{\chi}(u) \text { and } u \rightarrow T_{-\bar{\chi}}(u)
$$

of $S U(2)$ are identical. Hence, $S$ is an invariant scalar product for the representation of $S U(2)$ contained in $\chi$.

The functions ${ }^{5} D_{m n}^{J}(u)$ are therefore bases for the spaces $B_{z}$ and $B_{-\bar{\chi}}$ in the sense that

if $D_{J, m n}^{-\bar{x}} \in B_{-\bar{x}}, D_{J^{\prime}, m^{\prime} m^{\prime}}^{\chi} \in B_{\chi}$.

$$
S\left(D_{J, m n}^{-\bar{z}}(u), D_{J^{\prime}, m^{\prime} n^{\prime}}^{\chi}(u)\right)=\delta_{J J^{\prime}} \delta_{m m^{\prime}} \delta_{n n^{\prime}}
$$

One can also define matrix elements

$$
T_{J J^{\prime}, m m^{\prime}, n n^{\prime}}^{\chi}(g)=S\left(D_{J, m n}^{-\bar{x}}(u), T(g) D_{J^{\prime}, m^{\prime} n^{\prime}}^{\chi}(u)\right) .
$$

S. Strom has studied these matrix elements for the case of a unitary representation of the principal series ${ }^{6}$, (in this case $-\bar{\chi}=\chi$, hence $S$ is a scalar product in the space $B_{x}$ ).

A very important decomposition ${ }^{7}$ of elements of $S L(2 C)$ is the decomposition into generalized Euler angles.

Let

$$
\begin{aligned}
\Lambda_{i} & =\left\{\left(\begin{array}{cc}
\lambda_{i} & 0 \\
0 & \lambda_{i}-1
\end{array}\right)\right\} \\
\Phi & =\left\{\left(\begin{array}{ll}
c & s \\
s & c
\end{array}\right)\right\}
\end{aligned}
$$

where $\lambda_{i}, c, s \in \mathbb{C}$ and $c^{2}-s^{2}=1$. Then almost every ${ }^{7} \Sigma \in S L(2 C)$ can

4 Up to equivalence the representation depends only on $\chi$, not on the realization of the representation.

${ }^{5}$ By $D_{m, n}^{J}(u)$ we mean the $m, n$th matrix element in the representation $J$ of the group $S U(2)$, viewed as a function of $u \in S U(2)$. By $D_{J, m, n}^{\chi}(u)$ we mean the function $D_{m, n}^{J}(u)$ viewed as an element of the space $B_{\chi}$.

${ }^{6}$ See references [12], [13].

7 This decomposition is valid except on a subset of lower dimension. That is, we do not use this form when $c=0$ or $s=0$ in equation (5.6). 
be written

$$
\Sigma=\left(\begin{array}{ll}
\alpha & \beta \\
\gamma & \delta
\end{array}\right)=\Lambda_{1} \Phi \Lambda_{2}=\left(\begin{array}{ll}
\lambda_{1} \lambda_{2} c & \frac{\lambda_{1} s}{\lambda_{2}} \\
\frac{\lambda_{2} s}{\lambda_{1}} & \frac{c}{\lambda_{1} \lambda_{2}}
\end{array}\right) .
$$

It can be shown that for the subgroup $\Phi$, the properties (2) and (4) of the representation

$$
\chi=\left(n_{1}, n_{2}\right)=\left(\frac{\varrho+n}{2}, \frac{\varrho-n}{2}\right)=\langle\varrho, n\rangle,
$$

for any $\chi$ - not only those for which the representation is unitary together with the form of the functional $S$ imply that, asymptotically as $c \rightarrow \infty$, for any $f \in D_{\chi}, g \in D_{-\bar{\chi}}$

$$
S\left(g, T_{\chi}(\Phi) f\right) \underset{c \rightarrow \infty}{\longrightarrow}|c|^{-\varrho-2} \Phi_{1}+|c|^{+e-2} \Phi_{2}
$$

where $\Phi_{1}$ and $\Phi_{2}$ are bounded functions of $c$ which depend on $f$ and $g$.

A detailed discussion of this result will be given in the next paper ${ }^{8}$.

\section{Irreducible representations of the translation group in four complex dimensions}

The translation subgroup $T$ of CILG has irreducible representations labelled by two complex matrices $P, Q \in G L(2 C)$. The representation is defined by

$$
\begin{aligned}
T(X)|P, Q\rangle= & e^{i \frac{1}{2} \operatorname{Tr}[X P+\overline{X Q}]}|P, Q\rangle \\
& =e^{i\left[\left(X_{\mu}^{1}+i X_{\mu}^{2}\right)\left(P_{\mu}^{1}+i P_{\mu}^{2}\right)+\left(X_{\mu}^{1}-i X_{\mu}^{2}\right)\left(Q_{\mu}^{1}-i Q_{\mu}^{2}\right)\right]}|P, Q\rangle .
\end{aligned}
$$

This becomes an SLS representation, $D_{P, Q}$, if for each space

$$
D_{P, Q}^{1}=\{\alpha|P, Q\rangle: \alpha \in \mathbb{C}\}
$$

we take as the dual space

with the inner product

$$
D_{P, Q}^{2}=\{\beta|Q, P\rangle: \beta \in \mathbb{C}\}
$$

$$
(\beta|Q, P\rangle, \alpha|P, Q\rangle)=\bar{\beta} \alpha
$$

When $P=Q$, the representation is an irreducible unitary representation in one dimensional Hilbert space.

\section{Unitary and non-unitary representations of the complex inhomo- geneous Lorentz group}

To construct induced SLS representations of CILG we must compute the action of CILG on the space $\tilde{T}$ of irreducible representations of the (abelian, normal) subgroup of translations, find the orbits under this action, and the stationary group of some point on each orbit.

8 The result is derived in reference [9]. We comment on the significance in Section IX, as well as in references [9] and [10]. 
(Given a point $x$ on a $G$-space $X$, an orbit of $x$ is the subspace of $X$ consisting of all points into which the point $x$ can be mapped by the action. It is obviously a homogeneous space. The stationary group of $x$ is the subgroup of $G$ for which the action maps $x$ into itself. On the space $\widetilde{T}$, the translations generate the identity mapping on the entire space. Hence it is useful to define the little group of $x$ to be the stationary group of $x$ modulo the subgroup of translations.)

The action $\Phi$ of CILG on $T$ is defined by

$$
\begin{gathered}
\Phi[(X, \Sigma, \Lambda),|P, Q\rangle]=\left|\Lambda^{*} P \Sigma, \Lambda^{*} Q \Sigma\right\rangle \\
(X, \Sigma, \Lambda) \in \mathrm{CILG},|P, Q\rangle \in \tilde{T} .
\end{gathered}
$$

Observe that under this action the SLS representation $D_{P, Q}$ is mapped into the SLS representation $D_{\Lambda^{*} P \Sigma, \Lambda^{*} Q \Sigma}$.

The invariants of an orbit are

$$
M_{P}^{2}=\frac{1}{2} \operatorname{Tr}\left(P P_{+}\right), M_{Q}^{2}=\frac{1}{2} \operatorname{Tr}\left(Q Q_{+}\right), C=\frac{1}{2} \operatorname{Tr}\left(P Q_{+}\right) .
$$

We now tabulate the different types of orbits.

I. $P=Q=0$.

II. $P=0$, or $Q=0$, or $P=\lambda Q, \lambda \in C$.

Let $P \neq 0$ (the other case is equivalent). Then there are two subtypes

(a) $M_{P}=0$,

(b) $M_{P} \neq 0$.

III. $P \neq 0$, and $Q \neq 0$, and $P \neq \lambda Q$,

(a) $M_{P} \neq 0$, and $M_{Q} \neq 0$,

(b) $M_{P}=0$.

Case I. $P=Q=0$.

In this case the translations are represented by the identity operator, so the representation of CILG reduces to the representation of the subgroup of elements of the form

$$
(0, \Sigma, \Lambda)\left(0, \Sigma^{\prime}, \Lambda^{\prime}\right)=\left(0, \Sigma \Sigma^{\prime}, \Lambda \Lambda^{\prime}\right)
$$

which is isomorphic to $S L(2 C) \otimes S L(2 C)$.

The irreducible representations are therefore given by the outer product of two representations of $S L(2 C)$.

Case $I I(a)$. The space $\widetilde{T}_{0}: P \neq 0, Q=0, M_{P}=0$ or the equivalent cases.

In this case the little group is the group 3 . To see this we note that the matrix

$$
\left(\begin{array}{ll}
1 & 0 \\
0 & 0
\end{array}\right)
$$


is a point of this space. The action of CILG on this point is

$$
\left(\begin{array}{ll}
1 & 0 \\
0 & 0
\end{array}\right) \rightarrow \Lambda^{*}\left(\begin{array}{ll}
1 & 0 \\
0 & 0
\end{array}\right) \Sigma
$$

The equation

is satisfied when

$$
\left(\begin{array}{ll}
1 & 0 \\
0 & 0
\end{array}\right)=\Lambda^{*}\left(\begin{array}{ll}
1 & 0 \\
0 & 0
\end{array}\right) \Sigma
$$

$$
\Sigma=\left(\begin{array}{cc}
\lambda & 0 \\
z & \lambda-1
\end{array}\right) \quad \Lambda=\left(\begin{array}{ll}
\bar{\lambda}-1 & 0 \\
\bar{\omega} & \bar{\lambda}
\end{array}\right) .
$$

Thus, an element of the little group 8 is a pair of matrices

$$
\left[\left(\begin{array}{cc}
\lambda & 0 \\
z & \lambda^{-1}
\end{array}\right),\left(\begin{array}{ll}
\bar{\lambda}-1 & 0 \\
\bar{\omega} & \bar{\lambda}
\end{array}\right)\right]=(\lambda, z, \omega)
$$

with the multiplication law (4.2).

The representation is defined on functions

$$
f: \mathrm{CILG} \rightarrow D
$$

where $D$ is an SLS functional representation space of $S_{8, T} \subset$ CILG, the semidirect product of $B$ and the translation group. With respect to the translation group, $D$ is precisely that representation whose stationary group is $S_{3, T}$. That is, the functions $f$ satisfy

$$
\begin{aligned}
f(X \cdot \xi) & =T_{0}(X) f(\xi) \\
f(\zeta \cdot \xi) & =T_{8}(\zeta) f(\xi)
\end{aligned}
$$

where $X$ is a translation, $\xi \in \mathrm{CILG} \zeta \in \mathcal{B}$ and

$$
T_{0}(X) f(\xi)=e^{i \frac{1}{2} \operatorname{Tr}\left[\overline{\left.\left(\begin{array}{ll}
1 & 0 \\
0 & 0
\end{array}\right) X\right]}\right.} f_{1}(\xi) \otimes e^{\left.i \frac{1}{2} \operatorname{Tr}\left[\begin{array}{ll}
1 & 0 \\
0 & 0
\end{array}\right) X\right]} f_{2}(\xi)
$$

is an SLS representation of the translation group,

$$
T_{8}(\zeta) f(\xi)=f_{\zeta}(\xi)^{9}
$$

is an SLS function space representation of 8 of the type discussed in IV.

The representation of CILG is defined by

$$
T\left(\xi^{\prime}\right) f(\xi)=f\left(\xi \xi^{\prime}\right) .
$$

Case II (b). $\widetilde{T}_{M}: P \neq 0, M_{P} \neq 0, Q=0$ or the equivalent cases.

We use the second realization of CILG

$$
\begin{aligned}
\left(X^{\prime}, \Gamma^{\prime}, \Pi^{\prime}\right)\left(X^{\prime \prime}, \Gamma^{\prime \prime}, \Pi^{\prime \prime}\right) & =(X, \Gamma, \Pi) \\
X & =X^{\prime}+\Gamma^{\prime} \Pi^{\prime *-1} X^{\prime \prime} \Pi^{\prime *} \\
\Gamma & =\Gamma^{\prime} \Pi^{\prime *-1} \Gamma^{\prime \prime} \Pi^{\prime *} \\
\Pi & =\Pi \Pi^{\prime}
\end{aligned}
$$

9 The subscript notation is defined in equation 2.8. The variable on which $f$ depends, as an element of the space $D$, has been suppressed. 
The action of CILG on the point

$$
P_{0}=M 1
$$

is

Hence the subgroup

$$
1 \rightarrow \Pi * 1 \Gamma \Pi^{*-1} .
$$

$$
\{(0,1, \Pi)\}
$$

is the little group. Denoting by $S$ the subgroup

we see that

$$
S=\{(X, 1, \Pi)\}
$$

$$
\mathrm{CILG} / S \cong(0, \Gamma, 1) \cong \tilde{T}_{M}
$$

We discuss in this case a few more details than we have before, since this is the most important case (and because the fact that $\widetilde{T}_{M}$ is isomorphic to a subgroup of CILG makes the description in this case very economical, hence pedagogically useful as an example of induced representations).

Any element

can be factorized

$$
(X, \Gamma, \Pi) \in \mathrm{CILG}
$$

$$
(X, \Gamma, \Pi)=(X, 1, \Pi)\left(0, \Pi * \Gamma \Pi \Pi^{*-1}, 1\right) .
$$

Let $D_{S}$ denote an SLS representation of $S$. We make this into an SLS function space representation of CILG.

Consider functions

which have the property

$$
f: \mathrm{CILG} \rightarrow D_{S}
$$

where

$$
f(X, \Gamma, \Pi)=T_{M, x}(X, 1, \Pi) f\left(0, \Pi^{*} \Gamma \Pi^{*-1}, 1\right)
$$

$$
T_{M, \chi}(X, 1, \Pi) f=e^{i \bar{M} \frac{1}{2} \operatorname{Tr} \bar{X}} T_{-\bar{\chi}}(\Pi) f_{1} \otimes e^{i M \frac{1}{2} \operatorname{Tr} X} T_{\chi}(\Pi) f_{2}
$$

and $T_{\chi}(\Pi)$ is an irreducible representation of $S L(2 C)$.

For brevity we may write

and since

$$
f(0, \Gamma, 1)=f(\Gamma)
$$

we may also write

$$
\{(0, \Gamma, 1)\} \cong T_{M},
$$

$$
f(\Gamma)=f(P)
$$

where $P=M \Gamma$. (Equation (7.14b) defines $f(P)$.)

The representation is defined by

$$
\left[T\left(\xi^{\prime}\right) f\right](\xi)=f\left(\xi \xi^{\prime}\right) \quad \xi, \xi^{\prime} \in \mathrm{CILG} .
$$


Specifically, we have

$$
\begin{aligned}
T(X, \Gamma, \Pi) f\left(\Gamma^{\prime}\right) & =f\left[\left(0, \Gamma^{\prime}, 1\right)(X, \Gamma, \Pi)\right] \\
& =f\left[\left(\Gamma^{\prime} X, 1, \Pi\right)\left(0, \Pi^{*} \Gamma^{\prime} \Gamma \Pi^{*-1}, 1\right)\right] \\
& =T\left(\Gamma^{\prime} X, 1, \Pi\right) f\left(\Pi * \Gamma^{\prime} \Gamma \Pi^{*-1}\right) .
\end{aligned}
$$

Remark. This representation should be defined on a set of functions $f\left(I^{\prime}\right)$ which have suitable integrability and asymptotic properties, so that it is closed under the action of the representation, and irreducible with an appropriate definition of irreducibility.

We will not go into questions of this kind for non-unitary representations. For those representations of $S$ in this case, and of $S_{8, T}$ in Case II, and $S L(2 C) \otimes S L(2 C)$ in Case I, which are unitary and irreducible, the induced representation is unitary and irreducible if the function space is chosen so as to be a direct integral Hilbert space. Indeed, by running through all unitary, irreducible representations of $S, S_{8}, T$, and $S L(2 C) \otimes$ $\otimes S L(2 C)$, all the unitary, irreducible representations of CILG may be obtained.

Case $I I I(a) . P \neq 0, M_{P} \neq 0, Q \neq 0, M_{Q} \neq 0, P \neq \lambda Q$.

We can set

and

$$
P=g_{P} \Gamma_{0}
$$

where

$g_{P} \in G L(2 C)=\left(\begin{array}{ll}M_{P} & 0 \\ 0 & M_{P}\end{array}\right) g_{Q} \in G L(2 C)=\left(\begin{array}{ll}\alpha+\beta & 0 \\ 0 & \alpha-\beta\end{array}\right) \quad \alpha \neq \pm \beta$.

Notice that

and

$$
M_{Q}^{2}=\alpha^{2}-\beta^{2}
$$

$$
\frac{1}{2} \operatorname{Tr} g_{P} g_{Q^{+}}=M_{P} \alpha
$$

are invariants which fix $g_{Q}$.

The action is

$$
\begin{aligned}
& P \rightarrow \Pi^{*} P \Gamma \Pi^{*-1} \\
& Q \rightarrow \Pi^{*} Q \Gamma \Pi^{*-1} .
\end{aligned}
$$

This can be represented as

$$
\begin{aligned}
& \Gamma_{0} \rightarrow \Pi^{*} \Gamma_{0} \Gamma \Pi^{*-1} \\
& \Pi_{0} \rightarrow \Pi_{0} \Pi .
\end{aligned}
$$

Remark. The analysis in this case is very similar to the solution of the problem of reducing the Kronecker product of two representations. 
For that reduction we would proceed to "Fourier" analyze a function of $\Gamma_{0}$ and $\Pi_{0}$ with respect to $\Pi_{0}$ in the representations of $S L(2 C)$.

Equations (7.17) show that the little group in Case III (a) is the diagonal subgroup $\Lambda=\left(\begin{array}{rr}\lambda & 0 \\ 0 & \lambda^{-1}\end{array}\right) \lambda \in \mathbb{C}$, discussed in Section II (a).

We define

$$
\langle X, \Pi, \Gamma\rangle=(X, 1, \Pi)(0, \Gamma, 1)=\left(X, \Pi^{*-1} \Gamma \Pi^{*}, \Pi\right)
$$

and recall that $\Pi$ has a canonical decomposition given by equation 5.7

$$
\Pi=\Lambda_{1} \Phi \Lambda_{2}
$$

where $\Lambda_{1}$ and $\Lambda_{2}$ are diagonal matrices and $\Phi$ is of the form

$$
\Phi=\left(\begin{array}{ll}
c & s \\
s & c
\end{array}\right), \quad c^{2}-s^{2}=1 .
$$

Then the representation is defined by

$$
T(\xi) f\left(\xi^{\prime}\right)=f\left(\xi \xi^{\prime}\right)
$$

where

is defined by

$$
f: \mathrm{CILG} \rightarrow D_{S}
$$

and

$$
f(\langle X, \Pi, \Gamma\rangle)=T_{g, x}(X, \Lambda, 1) f\left(\left\langle 0, \Phi \Lambda_{2}, \Gamma\right\rangle\right)
$$

$$
T_{g, \chi}(X, \Lambda, 1) f=e^{i \frac{1}{2} \operatorname{Tr}\left[\overline{g_{P} X}+g_{Q} X\right]} T_{-\bar{\chi}}\left(\Lambda_{1}\right) f_{1} \otimes e^{i \frac{1}{2} \operatorname{Tr}\left[g_{P} X+\overline{\left.g_{Q} X\right]}\right.} T_{\chi}\left(\Lambda_{1}\right) f_{2}
$$

(here $\chi$ denotes an irreducible representation of the multiplicative group of complex numbers).

Notice that

$$
T(X, 1,1) f(\langle 0, \Pi, \Gamma\rangle)=f\left[\left(\Pi^{*-1} \Gamma X \Pi^{*}, 1,1\right)\langle 0, \Pi, \Gamma\rangle\right]
$$

which induces the correct action on $P$ and $Q$.

Case III (b). $P \neq 0, Q \neq 0, P \neq \lambda Q, M_{P}=0$.

We will not dicuss these cases in detail. There are two cases:

$$
\frac{1}{2} \operatorname{Tr} P Q_{+}=0 \text { and } \frac{1}{2} \operatorname{Tr} P Q_{+} \neq 0 .
$$

In the first case, the little group is the additive group of complex numbers. In the second case it is the multiplicative group of complex numbers. The reader should have no difficulty verifying this and constructing the representations.

\section{The Poincaré group}

In this section we will not discuss the construction of representations as the procedure is essentially the same as in the preceding sections. We will, however, list the different types of representations that occur. 
We first observe that the representations of the real translation subgroup have the form

$$
\begin{aligned}
& T\left(X^{1}\right)|P, Q\rangle=e^{i \frac{1}{2} \operatorname{Tr}\left(X^{1} P+\overline{\left.X^{1} Q\right)}\right.}|P, Q\rangle \quad P, Q \in G L(2 C) \\
& X^{1} \text { Hermitian. }
\end{aligned}
$$

Since $X^{1}$ is Hermitian, the argument of the trace can be put in the form

where

$$
X^{1}\left(P_{1}+i P_{2}\right) \quad P_{1}, P_{2} \text { Hermitian, }
$$

$$
\begin{aligned}
P_{1} & =P_{h}+Q_{h} \\
i P_{2} & =P_{a}-Q_{a}
\end{aligned}
$$

where $h$ and $a$ denote the Hermitian and anti-Hermitian parts respectively.

Under the action

$$
P_{1}+i P_{2} \rightarrow \Lambda^{*}\left(P_{1}+i P_{2}\right) \Lambda
$$

\begin{tabular}{|c|c|c|c|c|}
\hline & $M_{P_{1}} M_{P_{2}}$ & Extra conditions & $\left|\frac{1}{2} \operatorname{Tr}\left(P_{1} P_{2}+\right)\right|$ & Little Group \\
\hline 1. & $>0>0$ & $\begin{array}{l}P_{1}=\lambda P_{2} \\
P_{1} \neq \lambda P_{2}\end{array}$ & $\begin{aligned} & \sqrt{M_{P_{1}}^{2} M_{P_{2}}^{2}} \\
> & \sqrt{M_{P_{1}}^{2} M_{P_{2}}^{2}}\end{aligned}$ & $\begin{array}{l}S U(2) \\
U(1)\end{array}$ \\
\hline 2. & $\begin{array}{l}>0=0 \\
\quad \text { or v.v. }\end{array}$ & $\begin{array}{l}P_{2}=0 \\
P_{2} \neq 0\end{array}$ & $\begin{array}{r}0 \\
>0\end{array}$ & $\begin{array}{l}S U(2) \\
U(1)\end{array}$ \\
\hline 3. & $\begin{array}{l}>0<0 \\
\quad \text { or } \mathrm{v} . \mathrm{v} .\end{array}$ & all cases equivalent & $\geqq 0$ & $U(1)$ \\
\hline 4. & $=0=0$ & 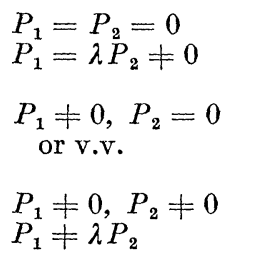 & $>0$ & $\begin{array}{l}S L(2 C) \\
\text { Euclidean group } \\
\text { of the plane } \\
\text { Euclidean group } \\
\text { of the plane } \\
U(1)\end{array}$ \\
\hline 5. & $\begin{array}{c}<0=0 \\
\text { or v.v. }\end{array}$ & $\begin{array}{l}P_{2}=0 \\
P_{2} \neq 0 \\
P_{2} \neq 0\end{array}$ & $\begin{array}{r}0 \\
0 \\
>0\end{array}$ & $\begin{array}{l}S L(2 R) \\
\text { Additive group } \\
\quad \text { of real numbers } \\
U(1)\end{array}$ \\
\hline 6. & $<0<0$ & $\begin{array}{l}P_{1}=\lambda P_{2} \\
P_{1} \neq \lambda P_{2} \\
P_{1} \neq \lambda P_{2}\end{array}$ & $\begin{aligned} & \sqrt{ } \sqrt{M_{P_{1}}^{2} M_{P_{2}}^{2}} \\
> & \sqrt{M_{P_{1}}^{2} M_{P_{2}}^{2}} \\
< & \sqrt{M_{P_{1}}^{2} M_{P_{2}}^{2}}\end{aligned}$ & $\begin{array}{l}S L(2 R) \\
U(1) \\
\text { Additive group } \\
\text { of real numbers }\end{array}$ \\
\hline
\end{tabular}

$P_{1}$ and $P_{2}$ transform separately. Hence the invariants are

$$
\frac{1}{2} \operatorname{Tr}\left(P_{1} P_{1}+\right)=\left(M_{1}\right)^{2}, \frac{1}{2} \operatorname{Tr}\left(P_{1} P_{2}+\right), \frac{1}{2} \operatorname{Tr}\left(P_{2} P_{2}+\right)=\left(M_{2}\right)^{2} .
$$

We now list the different cases and the little group for each case.

18 Commun. math. Phys., Vol. 4 
We now discuss the question of how an irreducible representation of CILG decomposes under the Poincaré group.

The Poincaré group has the form

$$
(X, \Lambda, \Lambda)\left(X^{\prime}, \Lambda^{\prime}, \Lambda^{\prime}\right)=\left(X+\Lambda X^{\prime} \Lambda^{*}, \Lambda \Lambda^{\prime}, \Lambda \Lambda^{\prime}\right)
$$

or

$$
\left(X, \Lambda \Lambda^{*}, \Lambda\right)\left(X^{\prime}, \Lambda^{\prime} \Lambda^{*}, \Lambda^{\prime}\right)=\left(X+\Lambda X^{\prime} \Lambda^{*}, \Lambda \Lambda^{\prime}\left(\Lambda \Lambda^{\prime}\right)^{*}, \Lambda \Lambda^{\prime}\right)
$$

where $X$ is Hermitian, and $\Lambda \in S L(2 C)$.

Recall the form of the states of a representation. There is a subgroup $H$, consisting of the translations and the little group, an SLS $D_{H}$, and a representation

of $H$ on $D_{H}$.

$$
h \rightarrow T_{H}(h) \quad h \in H
$$

The space of representations of CILG consists of functions

which satisfy

$$
f: \mathrm{CILG} \rightarrow D_{H}
$$

$$
f(h \xi)=T_{I I}(h) f(\xi) \quad h \in H, \xi \in \mathrm{CILG} .
$$

Let $\widetilde{K}=\mathrm{CILG} / H$. Let $K$ be a subset of CILG consisting of suitably chosen representations of cosets. $\widetilde{K} \cong K \subset$ CILG. Let $X \in \mathscr{P}$ be a translation.

Then for each $k \in K$, a representation of $X$ is defined by

$$
X \rightarrow T(X, 1,1) f(k)=T_{H}(k X) f(k)
$$

$T_{H}$ being the representation in $D_{H}$. $H$.

Let $\widetilde{T}_{K, H}$ denote the space of all such representations for given $K$ and

Then $\mathscr{P}$ induces an action on the space $\widetilde{T}_{K, H}$ given by

i.e. $k \rightarrow k^{\prime}$.

$$
T(p) f(k)=f(k p)=T_{H}\left(k p k^{\prime-1}\right) f\left(k^{\prime}\right)
$$

Each $H, \mathscr{P}$ double coset in $H \backslash \mathrm{CILG} / \mathscr{P}$ remains invariant under this action. (An $H_{1}, H_{2}$ double coset, where $H_{1}$ and $H_{2}$ are subgroups of a group $G$, is the set of all elements of the form $h_{1} g_{0} h_{2}$ where $g_{0}$ is a fixed element of $G$ and $h_{1}$ and $h_{2}$ run through $H_{1}$ and $H_{2}$ respectively. The space of $H_{1}, H_{2}$ double cosets is denoted $H_{1} \backslash G / H_{2}$ ).

Let $\sigma$ denote a double coset representative. Then $\sigma p \subset K$, where $p$ runs through $\mathscr{P}$, is the orbit of $\sigma$ under $\mathscr{P}$. Suppose that $\mathscr{N}_{\sigma} \subset \mathscr{P}$ is the stationary subgroup of $\mathscr{P}$ with respect to the representation (8.4) of $X$, with $k=\sigma$. That is, suppose that

for all $\eta \in \mathscr{N}_{\sigma}$.

$$
X \rightarrow T_{H}(\sigma X) \cong X \rightarrow T_{H}(\sigma \eta X)
$$


The representation of CILG is now completely decomposed by summing over the $H, \mathscr{P}$ double cosets, $\sigma$, and for each $\sigma$ summing over the irreducible representations of $\mathscr{N}_{\sigma}$ contained in the representation of CILG.

As an example, consider the representations

and

$$
f[(X, 1, \pi)(0, \Gamma, 1)]=e^{i M \frac{1}{2} \operatorname{Tr} X} f(\Gamma)
$$

$$
\begin{array}{r}
f[(X, 1, \pi)(0, \Gamma, 1)]=e^{i M \frac{1}{2} \operatorname{Re} \operatorname{Tr} X} f(\Gamma) \\
T\left(X^{\prime}, \Lambda \Lambda^{*}, \Lambda^{\prime}\right) f(\Gamma)=\left\{\begin{array}{l}
e^{i M \frac{1}{2} \operatorname{Tr}\left(\Gamma_{1}+i \Gamma_{2}\right) X^{\prime}} T_{\chi}(\Lambda) f\left(\Lambda^{*} \Gamma \Lambda\right) \\
e^{i M \frac{1}{2} \operatorname{Tr} \Gamma_{1} X^{\prime}} T_{\chi}(\Lambda) f\left(\Lambda^{*} \Gamma \Lambda\right)
\end{array}\right.
\end{array}
$$

Under the action, $\Gamma \rightarrow \Lambda^{*} \Gamma \Lambda$, the invariants are

$$
\alpha=\frac{1}{2} \operatorname{Tr} \Gamma_{1} \Gamma_{1}+, \beta=\frac{1}{2} \operatorname{Tr} \Gamma_{2} \Gamma_{2}+, \gamma=\frac{1}{2} \operatorname{Tr} \Gamma_{1} \Gamma_{2}+
$$

$\alpha, \beta, \gamma$ label the $\operatorname{cosets}^{10}$.

Given a particular coset, $C$, in case (a) each $\Gamma_{1}+i \Gamma_{2}$ belonging to that coset gives rise to a different representation of the translation subgroup. In case (b), every $\Gamma_{1}^{0}+i \Gamma_{2}$, for fixed $\Gamma_{1}^{0}$, gives rise to the same representation.

Hence, given a particular coset, in case (a) pick a particular $\Gamma_{1}^{0}+i \Gamma_{2}^{0}$ in that coset. Find the little group $L$ of this vector. Suppose $l \in L$. It is represented by

$$
f(\Gamma) \rightarrow T_{\chi}(l) f\left(l^{*} \Gamma l\right)
$$

Restricted to the $\operatorname{coset} C$, the representation of $\mathscr{P}$ reduces according to how the representation $\chi$ of $S L(2 C)$ contains irreducible representations $\lambda$ of $L C S L(2 C)$.

In case (b), given a particular coset, $C$, each $\Gamma_{1}+i \Gamma_{2}$ gives rise to a representation distinguished only by the value of $\Gamma_{1}$. Hence we pick a $\Gamma_{1}^{0} \in C$. Let $L_{\Gamma_{1}^{0}}$ denote the little group of this element.

It is represented by

$$
f(\Gamma) \rightarrow T_{\chi}(l) f\left(l^{*} \Gamma l\right), \quad l \in L_{\Gamma_{1}^{0}} .
$$

Under $L_{\Gamma_{1}^{0}}$ a representative $\Gamma_{1}^{0}+i \Gamma_{2}$ of the coset $C$ transforms by

$$
\Gamma_{1}^{0}+i \Gamma_{2} \rightarrow l^{*}\left(\Gamma_{1}^{0}+i \Gamma_{2}\right) l=\Gamma_{1}^{0}+i l^{*} \Gamma_{2} l .
$$

Hence, the reduction of the representation, in a particular coset, depends in general upon the reduction of $f$ as a function of $\Gamma_{2}$ as well as on the reduction of the representation $\chi$ of $S L(2 C)$ to $L \subset S L(2 C)$.

Of course, if $\Gamma_{2}$ is parallel to $\Gamma_{1}^{0}$ (as it is for the case $\Gamma_{2}=0$ ), then the reduction depends only on how the representation $\chi$ reduces $\left(f\left(\ldots \Gamma_{2}\right)\right.$ transforms as a constant).

${ }_{10}$ There are other invariants, such as the sign of the zero-th component of a time-like vector. 


\section{Physical significance}

CILG is a natural object to study in connection with the $S$-matrix as a function of the invariants, for all complex values of the invariants. We give a number of reasons:

(a) One can construct an $S$-matrix which is invariant under CILG as well as the Poincaré group. Restricted to physical values (for a given channel) of the invariants, the $S$-matrix is invariant under the restriction of CILG to its physical subgroup, the Poincaré group.

(b) One can perform a "partial wave analysis" on the $S$-matrix. This is performed on the $S$-matrix as a function of all complex values of the invariants (not just on $S$ as a function of the invariants in a physical region for a particular channel). The reduced $S$-matrix is interpreted as the matrix element of the $S$-operator restricted to the space of an irreducible representation of CILG. We write

$$
S \sim \int S_{\chi}(S) D_{\chi}\left(\cosh \frac{\theta}{2}\right) d \chi
$$

where $D_{z}\left(\cosh \frac{\theta}{2}\right)$ is a matrix element of the operator

$$
T_{\chi}\left(\begin{array}{ll}
\cosh \frac{\theta}{2} & \sinh \frac{\theta}{2} \\
\sinh \frac{\theta}{2} & \cosh \frac{\theta}{2}
\end{array}\right)
$$

in the SLS of the representation $\chi$ of $S L(2 C)$ (and $\cosh \theta$ as a function of $S$, and $T$, the Mandelstam variables, is just the scattering angle).

(c) Each term in 9.1 has a well defined asymptotic behavior as $\left|\cosh \frac{\theta}{2}\right| \rightarrow \infty$. This behavior is determined by $\chi$.

(d) The representations of CILG generalize the representations of $\mathscr{P}$. They allow unitary and non-unitary representations with "complex mass," (and perhaps illuminate some of the structure of the non-unitary representations of $\mathscr{P}$ with complex mass). They also allow both unitary and non-unitary representations of the little group, which (according to (b) and (c)) play part of the role of complex angular momentum for the $S$-matrix, and every such representation has a natural interpretation as a reducible representation of the Poincaré group.

(e) The analysis of the S-matrix, by means of CILG is a generalization of the method introduced by SERTorio and Toller ${ }^{11}$. The fact that non-unitary representations arise naturally for CILG (as long as the Poincaré subgroup is represented unitarily) as well as the fact that the representations of CILG have an immediate physical interpretation in the direct channel, are the most important reasons for considering CILG rather than the three dimensional Lorentz group.

${ }^{11}$ See references [11], [14], and [15]. 
We believe that the CILG will prove to be of value in studying the $S$-matrix. We plan to give a detailed discussion in another article, of the physical interpretation and some applications of CILG.

Acknowledgments. This work originated at Brandeis University and contains all the representation theoretic results of reference [9], and was completed at the Courant Institute of Mathematical Sciences. I would like to thank Dr. M. E. MAYER and Dr. S. Schweber for their constant encouragement and advice, and Dr. M. GRISARU for helpful conversations. I would like to thank Dr. E. SALETAN for making available to me, in advance of publication, his translation of reference [2]. I am grateful for the support of the National Science Foundation (NSF-GP-58) during the work at Brandeis, and to the National Science Foundation, Grant NSF-GP-3465, for support while at the Courant Institute of Mathematical Sciences.

\section{References}

[1] Feld, J. M. G.: Non-unitary dual spaces of groups. Acta Math. 114, 267 (1965).

[2] Gel'fand, I. M., M. I. Graev, and N. Ya. Vilenkin: Generalized functions, Vol. V. New York: Academic Press 1966. (English Translation).

[3] Joos, H.: Complex angular momentum and the representations of the Poincaré group with space-like momentum. Lectures in Theoretical Physics VII-A. Boulder: University of Colorado Press 1964.

[4] Mackey, G. W.: The theory of group representations. Lecture Notes, University of Chicago (1955).

[5] - Infinite dimensional group representations. Bull. Am. Math. Soc. 69, 628 (1963).

[6] MAYeR, M. E.: Representations of noncompact (locally compact) groups on rigged Hilbert spaces and their possible uses. Feldafing Seminar on Unified Theories of Elementary Particles, Proceedings, Max-PlanckInstitut für Physik und Astrophysik, Munich (1965).

[7] Moussa, P., and R. Stora: Some remarks on the product of irreducible representations of the inhomogeneous Lorentz group. Lectures in Theoretical Physics VII-A. Boulder: University of Colorado Press 1964.

[8] NaImaRK, M. A.: Linear representations of the Lorentz group. New York: Macmillan 1964. (English Translation).

[9] Roffman, E. H.: The complex inhomogeneous Lorentz group and complex angular momentum. Thesis submitted in Partial Fulfillment of the Degree Doctor of Philosophy, Brandeis University (1965).

[10] - The complex inhomogeneous Lorentz group and complex angular momentum. Phys. Rev. Letters 16, 210 (1966).

[11] Sertorio, L., and M. Toller: Complex angular momentum and the three dimensional Lorentz group. Nuovo Cimento 37, 631 (1965).

[12] Ströм, S.: On the matrix elements of a unitary representation of the homogeneous Lorentz group. Arkiv Fysik 29, 467 (1965).

[13] - A note on the matrix elements of a unitary representation of the homogeneous Lorentz group. Preprint, Chalmers University of Technology, Göteborg, Sweden.

[14] Toller, M.: Three dimensional Lorentz group and harmonic analysis of the scattering amplitude. Nuovo Cimento 37, 631 (1965).

[15] - Some consequences of a generalization of the Regge pole hypothesis. Preprint, Istituto di Fisica "G. Marconi", Universita di Roma.

[16] WigneR, E. P.: On unitary representations of the inhomogeneous Lorentz group. Ann. Math. 40, 149 (1939). 